\title{
PERFIL DAS NOTIFICAÇÕES DE CASOS DE SÍFILIS GESTACIONAL E SÍFILIS CONGÊNITA
}

PROFILE OF NOTIFICATIONS OF GESTATIONAL SYPHILIS AND CONGENITAL SYPHILIS

PERFIL DE LAS NOTIFICACIONES DE CASOS DE SÍFILIS GESTACIONAL Y SÍFILIS CONGÉNITA

Brena Geyse Mesquita Rocha Soares ${ }^{1}$

Maria Alana Duarte Marinho ${ }^{2}$

Maria Isabel Linhares 3

Dário da Silva Mota 4

\section{RESUMO}

Este estudo analisou o perfil das notificações de casos de sífilis congênita (SC) e sífilis gestacional (SG) em Sobral (CE),

Elevantados no Sistema de Informação de Agravos de Notificação (Sinan) para um período de 10 anos (2004-2013). A incidência anual média foi de 17,8 casos/100.000 habitantes. Observou-se um índice de maior ocorrência em mulheres com idades entre 20 e 39 anos; o nível de escolaridade das mulheres mais atingidas foi o Ensino Fundamental; e a zona urbana representou $86,8 \%$ dos casos. No periodo de estudo foram notificados 109 casos de SC, com maior taxa em 2012 (28,4\%). Com base na idade, houve predominância de 0 a 6 dias de vida - a denominada sífilis congênita precoce. De acordo com o número de parceiros da mãe, houve predomínio de indivíduos não tratados $(65,1 \%)$. Os achados deste estudo evidenciam que a sífilis ainda se encontra fora de controle em Sobral. Assim, há necessidade de implementar ações mais efetivas para o controle e a diminuição dos agravos causados aos pacientes.

Palavras-chave: Sífilis Congênita; Fatores de Risco; Estudos de Coorte.

\footnotetext{
1. Farmacêutica graduada pelo Instituto Superior de Teologia Aplicada (INTA). Residente em Neonatologia na Santa Casa de Misericórdia de Sobral (SCMS). Sobral (CE), Brasil.

2. Farmacêutica graduada pelo INTA. Residente em Neonatologia na SCMS. Sobral (CE), Brasil.

3. Farmacêutica graduada pela Universidade Federal do Ceará (UFC). Aluna de Mestrado em Farmacologia no INTA.

4. Enfermeiro graduado pela Universidade Estadual Vale do Acaraú (UVA). Residente em Neonatologia na SCMS.
} 


\section{ABSTRACT}

This study analyzed the profile of notifications of cases of congenital syphilis (CS) and gestational syphilis (GS) in Sobral, Ceará, Brazil, surveyed on the Brazilian Information System for Notification of Diseases (SINAN) for a 10-year period (2004-2013). The average annual incidence was 17.8 cases/100,000 inhabitants. A higher incidence rate was observed in women aged between 20 and 39 years; the school education level of the most affected women was Elementary School; and the urban area accounted for $86.8 \%$ of the cases. Within the study period, 109 cases of CS were notified, with the highest rate in 2012 (28.4\%). Having age as a basis, there was predominance from 0 to 6 days of life - the so-called early congenital syphilis. According to the number of mother's partners, there was predominance of untreated individuals (65.1\%). The findings of this study show that syphilis is still out of control in Sobral. Thus, there is a need for deploying more effective actions to control and reduce the damages caused to patients.

Keywords: Congenital Syphilis; Risk Factors; Cohort Studies.

\section{RESUMEN}

Este estudio analizó el perfil de las notificaciones de casos de sífilis congénita (SC) y sífilis gestacional (SG) en Sobral, Ceará, Brasil, encuestados en el Sistema Brasileño de Información para Notificación de Enfermedades (SINAN) por un período de 10 años (2004-2013). La incidencia anual promedio fue de 17,8 casos/100.000 habitantes. Se observó una tasa de incidencia más alta en mujeres entre 20 y 39 años; el nivel de educación escolar de las mujeres más afectadas fue la Enseñanza Primaria; y el área urbana representó $86,8 \%$ de los casos. Dentro del período de estudio, se notificaron 109 casos de SC, con la tasa más alta en 2012 (28,4\%). Teniendo la edad como base, hubo predominio de 0 a 6 días de vida - la llamada sífilis congénita temprana. De acuerdo con el número de parejas de la madre, hubo predominancia de individuos no tratados (65,1\%). Los hallazgos de este estudio muestran que la sífilis aún está fuera de control en Sobral. Así, hay necesidad de implementar acciones más efectivas para el control y la disminución de los agravios causados a los pacientes.

Palabras clave: Sífilis Congénita; Factores de Riesgo; Estudios de Cohorte.

\section{INTRODUÇÃO}

As doenças sexualmente transmissiveis (DST) são consideradas um dos mais comuns problemas de saúde pública em nível mundial. Nos países subdesenvolvidos, tais doenças representam as cinco principais causas de procura por serviços de saúde; as DST que podem ser transmitidas da mãe para o feto são as de maior preocupação, como, por exemplo, a sífilis - cuja prevalência estimada em mulheres grávidas varia de 1,4 a $2,8 \%$ no Brasil, com transmissão vertical em cerca de $25 \%$ dos pacientes ${ }^{1,2}$.

A sífilis é uma doença infectocontagiosa, causada pela bactéria espiroqueta Treponema pallidum; o tratamento é eficaz e de baixo custo, mas essa doença ainda é considerada um grave problema de saúde pública por sua alta prevalência ${ }^{3}$.

Trata-se de uma patologia que pode ocasionar muitos efeitos nocivos quando presente no período gestacional, caso não seja precocemente diagnosticada e tratada 4 .

Por meio da Portaria n. 542, de 22/12/1986, o Ministério da Saúde tornou a sífilis congênita (SC) uma doença de notificação compulsória para fins de vigilância epidemiológica no Brasil. Já a sífilis gestacional (SG) tornou-se uma doença de notificação compulsória por meio da Portaria n. 33, de $14 / 07 / 2005^{5}$.

Por meio do Sistema de Informação de Agravos de Notificação (Sinan), 80.041 casos de SC foram registrados no Brasil nos últimos 15 anos. A maioria desses casos se centrou nas regiões Sudeste $(45,9 \%)$ e Nordeste $(31,4 \%)^{3,6}$.

A SC resulta da disseminação/transmissão hematogênica, por via transplacentária, do T. pallidum da gestante infectada (não tratada ou inadequadamente tratada) para o concepto. 0 diagnóstico tardio pode acarretar maiores chances de contaminação para o feto e ocasionar maiores sequelas, portanto, deve-se evitar a contaminação vertical’.

A droga de escolha para tratamento é a penicilina $G$ benzatina intramuscular, com posologia de acordo com o estágio da doença ${ }^{8-10}$. Assim, estratégias inovadoras se mostram necessárias para garantir o diagnóstico precoce durante a gestação, possibilitando o tratamento antes do período mais efetivo para o feto - da $24^{\mathrm{a}}$ à $28^{\mathrm{a}}$ semana de gestação $0^{11}$.

Em Sobral, município do noroeste do Ceará, intensificouse a vigilância da SG a partir de 2006; esse monitoramento tardio da sífilis resultou em subnotificação ou sub-registro 
da doença na população masculina, que pode ter contribuído para a elevação do número de casos entre gestantes nos últimos anos e sua consequente transmissão vertical ${ }^{8,9}$.

Considerando a necessidade de diminuir a incidência de SC, mostra-se importante que os profissionais e os gestores da saúde tenham acesso às informações demonstrativas da magnitude do problema, para que disponham de subsídios para planejar novas intervenções e estratégias voltadas a diminuir os índices da enfermidade.

Esta pesquisa analisou o perfil epidemiológico dos casos notificados de SG e SC em Sobral.

\section{MÉTODOS}

Trata-se de pesquisa documental, descritiva e retrospectiva, com abordagem quantitativa, que analisa todos os casos notificados de SG e SC em Sobral no período de 2004 a 2013.

Foram avaliados os seguintes parâmetros: distribuição da doença por zona de residência; faixa etária; escolaridade; etnia; testes diagnósticos; evolução da doença; e idade da criança infectada.

0s dados obtidos foram tabulados e analisados por meio da estatística descritiva. 0s resultados são expressos em frequência relativa (porcentagem). 0s gráficos e as tabelas foram elaborados no programa computacional Microsoft Excel, versão Windows 8.

Por se tratar de estudo com dados secundários de livre acesso, não foi necessária a aprovação por um comitê de ética em pesquisa.

\section{RESULTADOS}

A Figura 1 indica que foram registrados 334 casos de SG em Sobral entre 2004 e 2013 . 0 número de casos manteve-se crescente, com apenas uma redução (2009). Os maiores números de casos ocorreram entre 2010 e 2012.

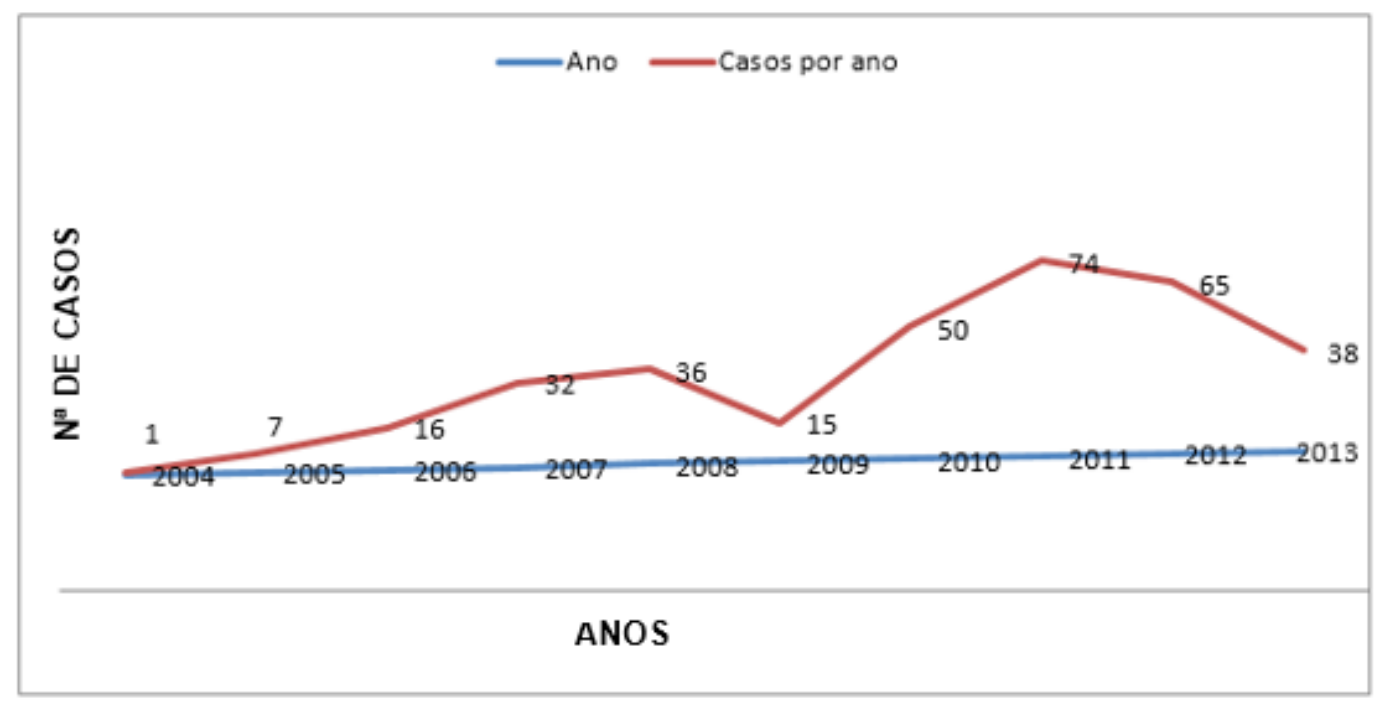

Figura 1. Distribuição dos casos de sífilis gestacional em Sobral (2004-2013).

Fonte: Elaborada pelos autores.

Outro estudo apresenta resultados semelhantes, com maior índice de casos entre 2008 e 2010 e menor incidência em $2006^{1}$.

A notificação de apenas 1 caso em 2004 e de 7 casos em 2005 levanta dúvidas quanto à ocorrência de sub-registro, devido à grande ascendência nos anos posteriores. A notificação adequada se mostra fundamental para o controle da sífilis, de modo que possibilite a investigação e o acompanhamento do caso. A falta de notificação também acarreta distorções no planejamento das ações de saúde por parte dos órgãos gestores nas 3 esferas de governo ${ }^{12-14}$.

A incidência anual média foi de 17,8 casos/100.000 habitantes no período em foco (Tabela 1 ). 
Tabela 1 - Média e incidência dos casos de sífilis gestacional em Sobral (2004-2013)

\begin{tabular}{lcc}
\hline Ano & Média & Incidência \\
\hline 2004 & 0,08 & 0,6 \\
2005 & 0,58 & 4,0 \\
2006 & 1,33 & 9,1 \\
2007 & 2,66 & 17,8 \\
2008 & 3,00 & 19,9 \\
2009 & 1,25 & 8,2 \\
2010 & 4,16 & 26,5 \\
2011 & 6,16 & 38,7 \\
2012 & 5,41 & 33,6 \\
2013 & 3,16 & 19,1 \\
\hline
\end{tabular}

Fonte: Elaborada pelos autores.

A maior incidência do agravo em Sobral ocorreu em 2011, com 38,7 casos $/ 100.000$ habitantes e a menor incidência ocorreu em 2004, com 0,6 casos/100.000 habitantes.

Com base na faixa etária, houve maior ocorrência entre mulheres dos 20 aos 39 anos $(75,7 \%)$.

Em relação à etnia, houve predomínio de pessoas pardas (Tabela 2).

Tabela 2 - Casos de sífilis gestacional em Sobral por faixa etária e etnia (2004-2013)

\begin{tabular}{lcc}
\hline Variável & N & \% \\
\hline Etnia & 5 & 1,5 \\
Ignorada/branca & 31 & 9,3 \\
Branca & 45 & 13,5 \\
Negra & 3 & 0,9 \\
Amarela & 250 & 74,8 \\
Parda & 334 & $100 \%$ \\
TOTAL & & \\
Faixa etária & 7 & 2,1 \\
$\leq 14$ & 67 & 20,1 \\
15-29 & 253 & 75,7 \\
20-39 & 7 & 2,1 \\
40-59 & 334 & $100 \%$ \\
\hline TOTAL & &
\end{tabular}

* $\mathrm{N}$ : número de casos.

* \%: número percentual de casos.

Fonte: Elaborada pelos autores.

Um estudo realizado no Rio de Janeiro e outro realizado no Distrito Federal também revelam a prevalência de SG em mulheres jovens (de 20 a 39 anos). Além disso, dados nacionais indicam que $53,5 \%$ dos casos notificados no Brasil se encontram na mesma faixa etária ${ }^{15-17}$.

Outra pesquisa, realizada no Piauí, corrobora os achados deste estudo: $64,4 \%$ das mulheres eram negras e pardas e
$29,6 \%$ eram brancas ${ }^{18}$.

Houve prevalência do agravo na zona urbana $(86,8 \%)$. Quanto ao nível de escolaridade, as mulheres mais atingidas em Sobral apresentaram da $5^{\mathrm{a}}$ à $8^{\mathrm{a}}$ série $(24,8 \%)$.

Tabela 3 - Casos de sífilis gestacional em Sobral por zona de residência e escolaridade (2004-2013)

\begin{tabular}{|c|c|c|}
\hline Variável & N & $\%$ \\
\hline \multicolumn{3}{|l|}{ Zona de residência } \\
\hline Rural & 36 & 10,8 \\
\hline Urbana & 290 & 86,8 \\
\hline Não Informada & 8 & 2,4 \\
\hline TOTAL & 334 & $100 \%$ \\
\hline \multicolumn{3}{|l|}{ Escolaridade } \\
\hline Ignorada & 82 & 24,5 \\
\hline Analfabeta & 7 & 2,1 \\
\hline $1^{\mathrm{a}}$ à $4^{\mathrm{a}}$ série & 41 & 12,3 \\
\hline $4^{\mathrm{a}}$ série completa & 33 & 9,9 \\
\hline $5^{\mathrm{a}}$ à $8^{\mathrm{a}}$ série & 83 & 24,8 \\
\hline Ensino Fundamental completo & 34 & 10,2 \\
\hline Ensino Médio incompleto & 26 & 7,8 \\
\hline Ensino Médio completo & 26 & 7,8 \\
\hline Ensino Superior incompleto & 1 & 0,3 \\
\hline Ensino Superior completo & 1 & 0,3 \\
\hline TOTAL & 334 & $100 \%$ \\
\hline
\end{tabular}

* $\mathrm{N}$ : número de casos.

* \%: número percentual de casos.

Fonte: Elaborada pelos autores.

Dados nacionais revelam essa mesma característica: $23 \%$ dos casos apresentam escolaridade da $5^{\mathrm{a}}$ a $8^{\mathrm{a}}$ série no Brasil em $2007^{17}$. A escolaridade parece ter menor destaque nas práticas de risco para as DST: atualmente, a população brasileira tem tido considerável acesso a informações básicas sobre as formas de transmissão de tais doenças. Entretanto, - acesso aos meios de prevenção e de tratamento está diretamente relacionado ao nível de escolaridade ${ }^{19}$.

A realização de testes treponêmicos e não treponêmicos nas gestantes portadoras de sífilis só foi notificada a partir de 2007. Esse fato evidencia uma falha no sistema de notificações, o que pode prejudicar os estudos epidemiológicos. A Figura 2 ilustra a realização dos testes treponêmicos. 


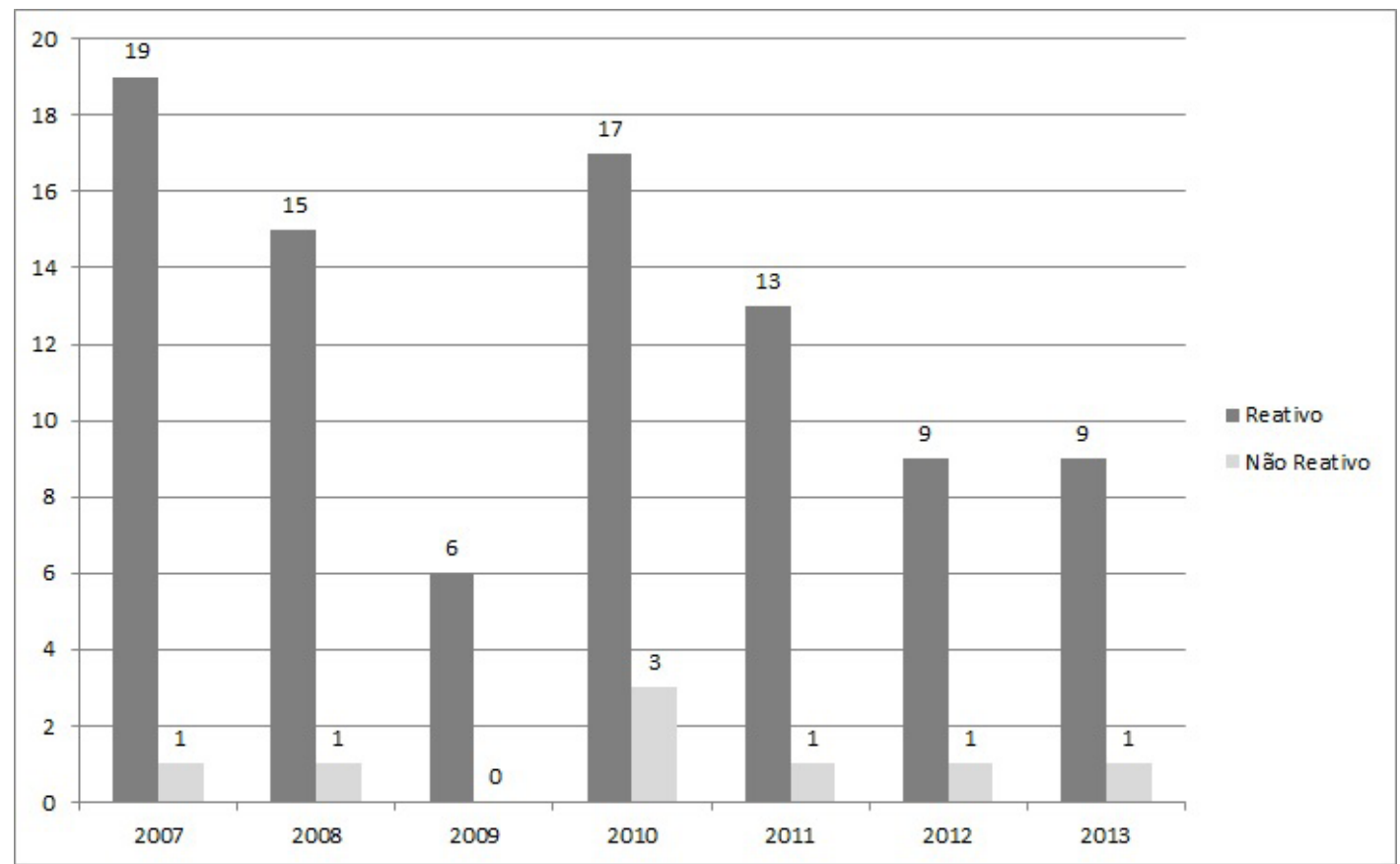

Figura 2. Distribuição dos casos de sífilis gestacional em Sobral segundo a realização dos testes treponêmicos (2004-2013). Fonte: Elaborada pelos autores.

A grande maioria das gestantes positivou em todos os anos nos testes treponêmicos (82,3\%); 190 gestantes não realizaram os testes durante o pré-natal e 24 mulheres ignoraram essa variável.

A reatividade nos testes treponêmicos indica que a pessoa teve contato com o T. pallidum em alguma época de sua vida e desenvolveu anticorpos específicos - o que confirma o diagnóstico ${ }^{20}$.

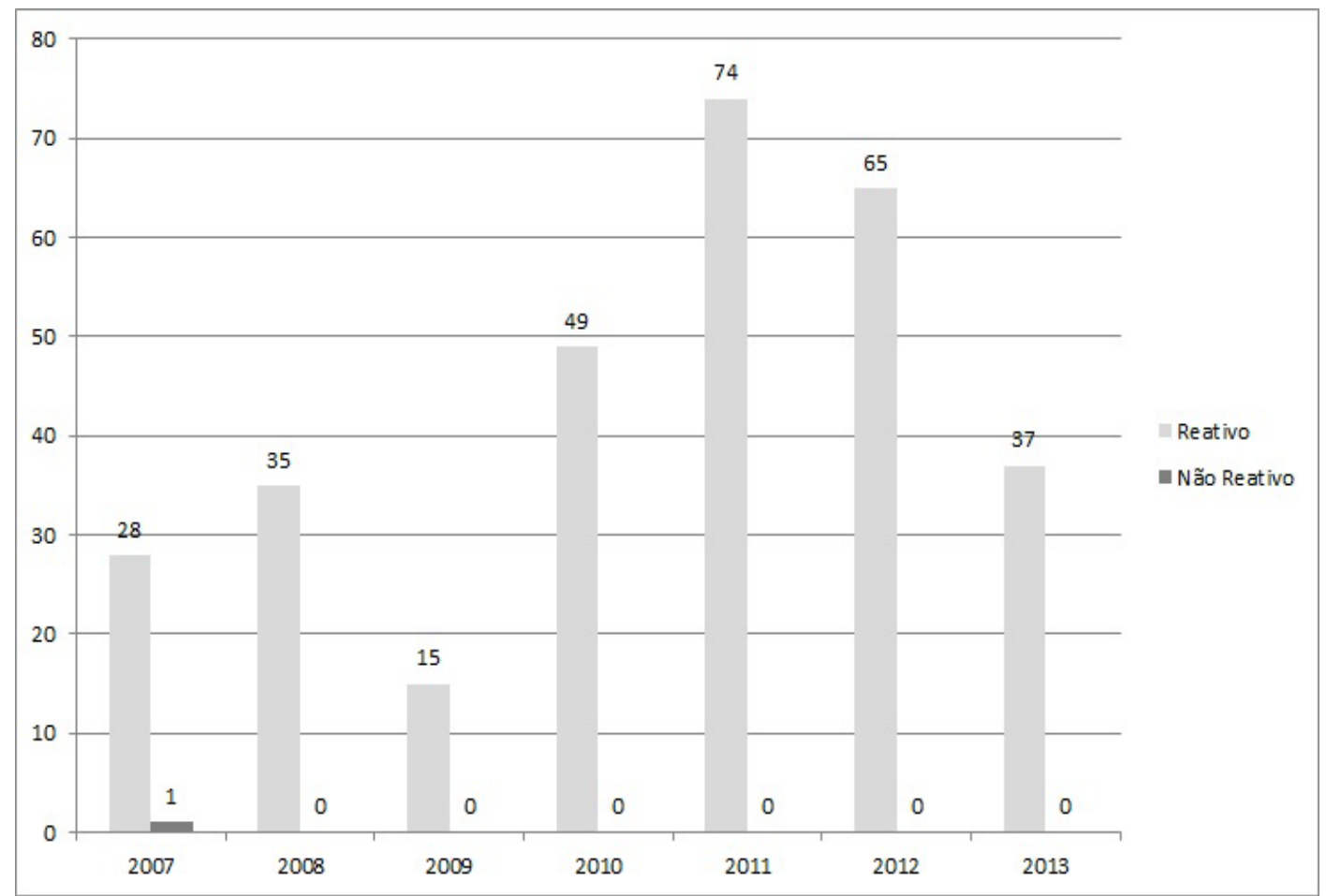

Figura 3. Distribuição dos casos de sífilis gestacional em Sobral segundo a realização dos testes não treponêmicos (2004-2013). Fonte: Elaborada pelos autores.

Dados do Ministério da Saúde mostram que 16\% das mulheres grávidas não realizaram os testes não treponêmicos antes ou depois da gravidez em 2012. Essa realidade nacional confirma a relação entre os casos de SC e as falhas na assistência pré-natal. 
Os testes não treponêmicos são mais utilizados no acompanhamento do tratamento, portanto, são utilizados para visualizar a evolução da doença e determinar se o tratamento é eficaz ${ }^{20}$.

A classificação clínica não foi preenchida em $100 \%$ das notificações, o que impossibilita a análise dos estágios da sífilis entre gestantes. 0 pré-natal foi realizado em $99 \%$ dos casos e $1 \%$ das mulheres ignorou a variável.

Apesar do crescimento do número de notificações, ainda se observa preenchimento incompleto ou incorreto de alguns campos da ficha de investigação, o que denota omissão ou banalização da notificação, que pode decorrer do desconhecimento epidemiológico acerca do agravo ou da ausência de foco na prevenção coletiva ${ }^{21}$.

\section{Sifilis congênita}

Entre janeiro de 1998 e junho de 2012 foram notificados 80.041 casos de SC no Brasil. A maioria dos casos se centrou nas regiões Sudeste e Nordeste ${ }^{22}$.

Em Sobral, a SC teve 109 casos notificados de 2004 a 2013. Não houve diferença significativa entre os sexos: 44\% do sexo masculino e $51,4 \%$ do sexo feminino (4,6\% das notificações ignoraram essa informação). Em 2004 não houve notificação de casos.

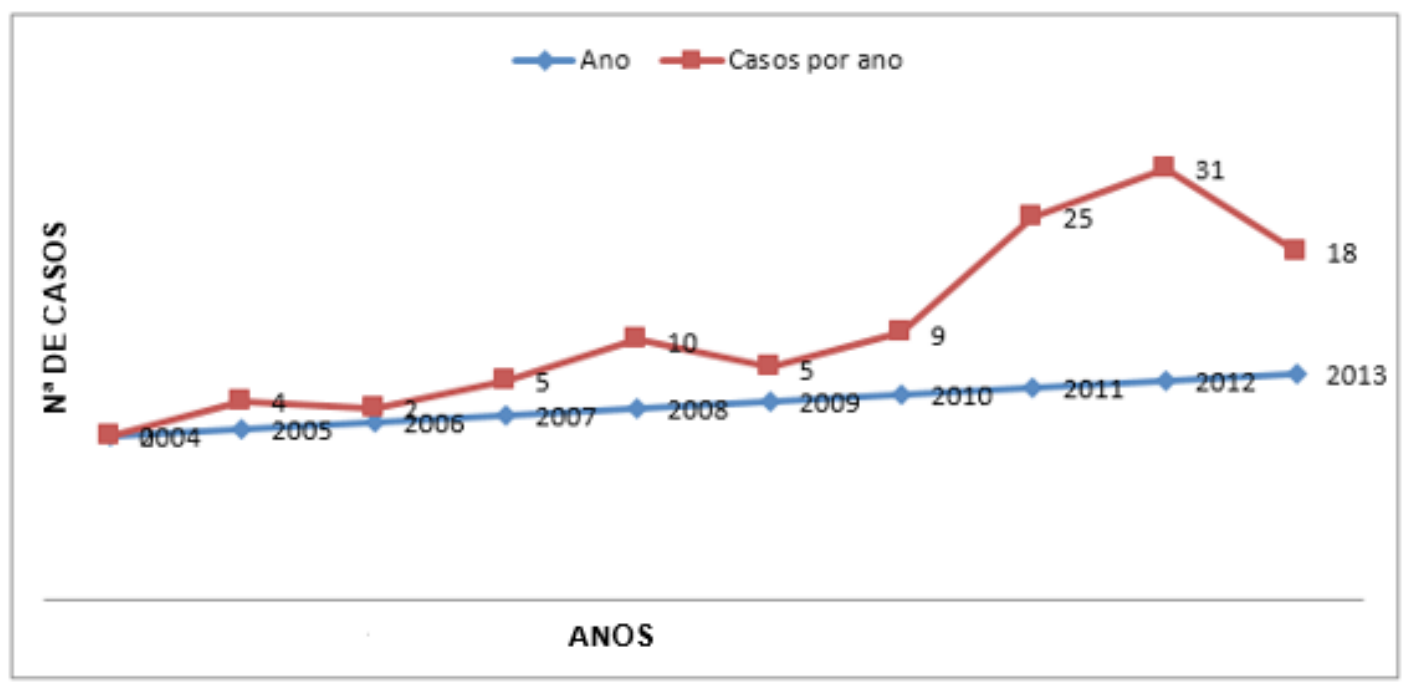

Figura 4. Distribuição dos casos de sífilis congênita em Sobral (2004-2013).

Fonte: Elaborada pelos autores.

Como ilustra a Figura 4, a SC apresentou maior índice em 2012 (28,4\%), seguido por $2011(22,9 \%)$ e 2013 (16,5\%). Nos anos anteriores foram registrados números menores de casos, o que levanta dúvidas quanto a possíveis subnotificações. Um estudo mostrou uma série histórica ascendente de 2001 a 2006 no Ceará ${ }^{10}$; e outro estudo identificou a ocorrência de 186 casos dessa doença de 2007 a $2009^{23}$.

Algumas metas foram estabelecidas pelo Pacto pela Vida para o biênio 2010-1011, selando compromisso entre os gestores do Sistema Único de Saúde (SUS) em torno de prioridades e indicadores que impactam as condições de saúde da população. 0s resultados esperados para SC no ano vigente apresentariam uma redução de $15 \%$ em comparação a 2007 . Sobral não conseguiu alcançar a meta estabelecida: foram notificados 9 casos (aumento de $80 \%$ ) ${ }^{1}$.

Com base na faixa etária, a Tabela 4 indica maior ocorrência de SC precoce $-97 \%$ dos casos foram de 0 a 6 dias de vida.

Tabela 4 - Casos de sífilis congênita em Sobral por sexo e faixa etária (2004-2013).

\begin{tabular}{lcc}
\hline Variável & $\mathbf{N}$ & $\%$ \\
\hline Sexo & & 4,6 \\
Ignorado & 5 & 44,0 \\
Masculino & 48 & 51,4 \\
Feminino & 56 & $100 \%$ \\
\hline TOTAL & 109 & \\
\hline
\end{tabular}




\begin{tabular}{lcc}
\hline Variável & N & \% \\
\hline \hline Faixa etária da criança & 106 & 97,3 \\
$0-6$ dias & 2 & 1,8 \\
$7-27$ dias & 1 & 0,9 \\
28 dias ou mais & 109 & $100 \%$ \\
TOTAL & \\
\hline
\end{tabular}

* N: número de casos.

* \%: número percentual de casos.

Fonte: Elaborada pelos autores.

Pesquisas mostraram que há maior ocorrência de SC de 0 a 6 dias em todo o Brasil, confirmando a predominância de sífilis precoce - o que favorece o tratamento ${ }^{23}$. A faixa etária deste estudo não contempla as mães, pois as notificações apresentam predominância de $\geq 80$ anos $(93,6 \%)$. Esses dados demonstram mais uma falha do sistema de notificação.

Observou-se que $97,2 \%$ das mães realizaram o pré-natal corretamente. Em relação à detecção de sífilis materna, os maiores índices foram detectados durante o pré-natal (61,5\% dos casos). Mesmo com a maioria das gestantes tendo realizado pré-natal de forma adequada e tendo sido diagnosticadas, houve muitos casos de transmissão vertical - o que indica uma grave falha na atenção pré-natal e na assistência à gestante.

Esse índice de casos de SC pressupõe que não houve tratamento adequado à gestante. Para que a gestante seja considerada adequadamente tratada, afastando a possibilidade de infecção neonatal, ela deve: receber a medicação na dosagem total e na quantidade de aplicação de acordo com o estadiamento da infecção; e ter finalizado o tratamento ao menos 30 dias antes do parto. Além disso, o parceiro deve ser tratado, concomitantemente, com o mesmo esquema ${ }^{24-26}$.

A Figura 5 ilustra que o número de parceiros não tratados foi maior $(65,1 \%$ dos casos) e que $11 \%$ das pessoas ignoraram essa variável.

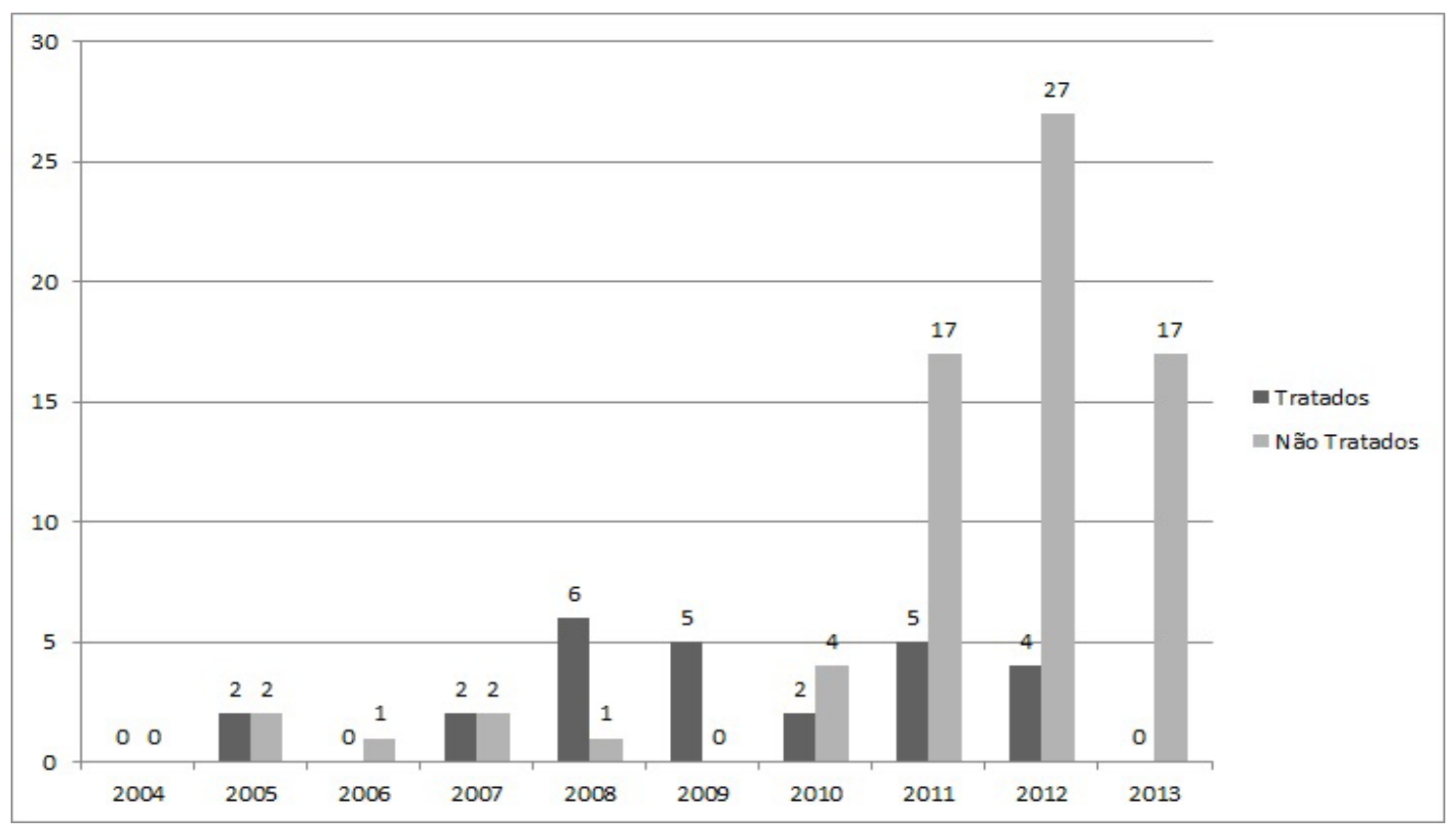

Figura 5. Distribuição dos casos de sífilis congênita em Sobral por sexo e quantidade de parceiros da mãe (2004-2013). Fonte: Elaborada pelos autores.

Diante desse fato, o controle da sífilis na gestação torna-se limitado, pois as gestantes infectadas vivenciam a ineficiência do tratamento, a reinfecção e a transmissão vertical. A ausência de tratamento adequado aumenta o risco de transmissão da sífilis da mãe para o concepto - que pode variar de $49 \%$ a $80 \%{ }^{18,26}$.

Este estudo corrobora pesquisas realizadas no Espírito Santo e no Amazonas, onde $66 \%$ e $67,1 \%$ dos parceiros não receberam tratamento, respectivamente. Diante disso, mostra-se necessária a comunicação e a busca ativa dos parceiros, que, em grande parte dos casos, não usam preservativos e não aderem ao tratamento. Tal situação, o não tratamento do parceiro, torna-se uma das grandes responsáveis pela reinfecção das gestantes e pela perpetuação e conservação dos casos de SC - mesmo que a gestante tenha recebido o tratamento adequado ${ }^{7,21,27}$. 
Assim, a inclusão do parceiro no pré-natal constitui relevante estratégia para a abordagem desse problema de saúde pública, uma medida categórica para a cura materna eficaz e, por conseguinte, para a eliminação do agravo².

Neste estudo, entretanto, $88,1 \%$ dos casos notificados evoluíram com a criança viva e apenas $1,8 \%$ dos casos levaram a óbito por SC, o que corrobora um estudo realizado no Piauí - no qual $89 \%$ dos casos evoluíram com as crianças vivas (que receberam tratamentos posteriores) ${ }^{18}$.

Alguns fatores influenciam o baixo índice de diagnósticos de SC durante o pré-natal, que pode estar relacionado ao baixo número de consultas pré-natal, à ausência do exame "venereal disease research laboratory" (VDRL) no primeiro e no terceiro trimestre gestacional, ao atraso na entrega dos resultados por parte dos laboratórios, à ausência de retorno da gestante com os resultados dos exames, bem como à falha do serviço na busca das gestantes que abandonam o acompanhamento pré-natal e, ainda, à adoção de tratamentos inadequados ${ }^{7}$.

\section{CONCLUSÃO}

Os achados deste estudo evidenciam que a SG, em combinação com a SC, ainda se encontra fora de controle em Sobral. Nos últimos anos, tem ocorrido significativa variação do número de casos, o que suscita a discussão de pontos relativos à assistência pré-natal e à prevenção da sífilis.

Diante disso, mostram-se necessárias ações mais significativas para o controle desse agravo. Ressalta-se a importância da educação em saúde com a população, com vistas a esclarecer os principais pontos relativos à doença.

\section{CONTRIBUIÇÃO DOS AUTORES}

Brena Geyse Mesquita Rocha Soares contribuiu com 0 delineamento e a realização da pesquisa; Maria Alana Duarte Marinho contribuiu com a redação e revisão do manuscrito; Maria Isabel Linhares contribuiu com a revisão crítica do manuscrito; Dário da Silva Mota contribuiu com a coleta de dados.

\section{REFERÊNCIAS}

1. Mesquita K0, Lima GK, Flôr SMC, Freitas CASL, Linhares MSC. Perfil epidemiológico dos casos de sífilis em gestante no município de Sobral, Ceará, de 2006 a 2010. Sanare (Sobral, Online) [serial on the internet]. 2012 [cited 2016 Nov 20];11(1):13-7. Available from: https://sanare.emnuvens. com.br/sanare/article/view/261

2. Magalhães DMS, Kawaguchi IAL, Dias A, Calderon IMP. Sífilis materna e congênita: ainda um desafio. Cad Saúde Pública [serial on the internet]. 2013 [cited 2016 Nov 20];29(6):1109-20. Available from: http://www.scielo.br/ $\mathrm{pdf} / \mathrm{csp} / \mathrm{v} 29 \mathrm{n} 6 / \mathrm{a} 08 \mathrm{v} 29 \mathrm{n} 6 . \mathrm{pdf}$
3. Matthes ACS, Lino APS, Costa CA, Mendonça CV, Bel DD. Sífilis congênita: mais de 500 anos de existência e ainda uma doença em vigência. Pediatr Mod [serial on the internet]. 2012 [cited 2016 Nov 20];48(4):149-54. Available from: http://www.moreirajr.com.br/revistas.asp?fase $=$ r003\&id materia $=4977$

4. Souza BC, Santana LS. As consequências da sífilis congênita no binômio materno-fetal: um estudo de revisão. Interfaces Científicas: Saúde e Ambiente [serial on the internet]. 2013 [cited 2016 Nov 20];1(3):59-67. Available from: $\quad$ https://periodicos.set.edu.br/index.php/saude/ article/view/746/440

5. Brasil. Avaliação da notificação da sífilis congênita no Brasil, 2000 a 2003. Boletim Epidemiológico Eletrônico [serial on the internet]. 2007 [cited 2016 Nov 20];7(7):15. Available from: http://portalsaude.saude.gov.br/images/ pdf/2014/julho/16/Ano07-n07-aval-notif-sifilis-cong-brcompleto--1-.pdf

6. Filippini FB, Krapf M0, Carli M, Liz FM, Zenil APD. Casos tratados de sífilis congênita no Hospital Geral de Caxias do Sul entre 2010 e 2013. Blucher Medical Proceedings [serial on the internet]. 2014 [cited 2016 Nov 20];1(5):26. Available from: http://www.proceedings.blucher.com.br/articledetails/casos-tratados-de-sfilis-congnita-no-hospital-geralde-caxias-do-sul-entre-2010-e-2013-13342

7. Lima MG, Santos RFR, Barbosa GJA, Ribeiro GS. Incidência e fatores de risco para sífilis congênita em Belo Horizonte, Minas Gerais, 2001-2008. Ciênc Saúde Coletiva [serial on the internet]. 2013 [cited 2016 Nov 20];18(2):499-506. Available from: http://www.scielo.br/pdf/csc/v18n2/21.pdf

8. Instituto Brasileiro de Geografia e Estatística. Ceará > Sobral [document on the internet]. 2016 [cited 2016 Jul 6]. Available from: http://www.cidades.ibge.gov.br/xtras/perfil. php?lang=\&codmun=231290\&search=ceara|sobral

9. Domingues RMSM, Leal MC. Incidência de sífilis congênita e fatores associados à transmissão vertical da sífilis: dados do estudo Nascer no Brasil. Cad Saúde Pública [serial on the internet]. 2016 [cited 2016 Nov 20];32(6):499-506. Available from: http://www.scielo.br/pdf/csp/v32n6/16784464-csp-32-06-e00082415.pdf

10. Ximenes IPE, Moura ERF, Freitas GL, Oliveira NC. Incidência e controle da sífilis congênita no Ceará. Rev RENE [serial on the internet]. 2008 [cited 20160 ct 9];9(3):74-80. Available from: http://www.redalyc.org/html/3240/324027963010/

11. França ISX, Batista JDL, Coura AS, Oliveira CF, Araújo $A K F$, Sousa FS. Fatores associados à notificação da sífilis congênita: um indicador de qualidade da assistência prénatal. Rev RENE [serial on the internet]. 2015 [cited 2016 Sep 10];16(3):374-81. Available from: http://repositorio. ufc.br/bitstream/riufc/14356/1/2015_art isxfranca.pdf 
12. Brasil. Portaria GM/MS n. 201, de 3 de novembro de 2010. Estabelece os parâmetros para monitoramento da regularidade na alimentação do Sinan e do SIM para fins de manutenção do repasse de recursos do Componente de Vigilância e Promoção da Saúde do Bloco de Vigilância em Saúde [document on the internet]. Brasília (DF): Ministério de Saúde; 2010 [cited 2016 Nov 20]. Available from: http://bvsms.saude.gov.br/ bvs/saudelegis/svs/2010/prt0201 0311 2010.html

13. Penna GO, Domingues CMAS, Siqueira Júnior JB, Elkhoury ANSM, Cechinel MP, Grossi MAF, et al. Dermatological diseases of compulsory notification in Brazil. An Bras Dermatol [serial on the internet]. 2011 [cited 2016 Nov 20];86(5):865-77. Available from: http://www.scielo.br/pdf/abd/v86n5/en v86n5a02.pdf

14. Silva GA, Oliveira CMG. 0 registro das doenças de notificação compulsória: a participação dos profissionais da saúde e da comunidade. Rev Epidemiol Controle Infecç [serial on the internet]. 2014 [cited 2016 Nov 20];4(3):21520. Available from: https://online.unisc.br/seer/index.php/ epidemiologia/article/view/4578

15. Saraceni V, Guimarães MHFS, Theme Filha MM, Leal MC. Mortalidade perinatal por sífilis congênita: indicador da qualidade da atenção à mulher e a criança. Cad Saúde Pública [serial on the internet]. 2005 [cited 2016 Nov 20];21(4):1244-50. Available from: http://www.scielo.br/ $\mathrm{pdf} / \mathrm{csp} / \mathrm{v} 21 \mathrm{n} 4 / 27 . \mathrm{pdf}$

16. Leitão EJL, Canedo MCM, Furiatti MF, Oliveira LRS, Diener LS, Lobo MP, et al. Sífilis gestacional como indicador de qualidade do pré-natal no Centro de Saúde Samambaia-DF. Comun Ciênc Saúde [serial on the internet]. 2009 [cited 2016 Nov 20];20(4):307-14. Available from: http://www.escs.edu. br/pesquisa/revista/2009Vol20 4sifilisgestacional.pdf

17. Brasil. Boletim Epidemiológico AIDS e DST. 2014;2(1).

18. Almeida PD, Araújo Filho ACA, Araújo AKL, Carvalho ML, Silva MGP, Araújo TME. Análise epidemiológica da sífilis congênita no Piauí. Revista Interdisciplinar [serial on the internet]. 2015 [cited 2016 Nov 20];8(1):62-70. Available from: http://revistainterdisciplinar.uninovafapi.edu.br/ index.php/revinter/article/view/453

19. Germano FN, Silva TMG, Mendoza-Sassi R, Martínez AMB. Alta prevalência de usuários que não retornam ao Centro de Testagem e Aconselhamento (CTA) para o conhecimento do seu status sorológico-Rio Grande, RS, Brasil. Ciênc Saúde Coletiva [serial on the internet]. 2008 [cited 2016 Nov 20];13(3):1033-40. Available from: http://www.scielo.br/ $\mathrm{pdf} / \mathrm{csc} / \mathrm{v} 13 \mathrm{n} 3 / 26 . \mathrm{pdf}$

20. Vaz AJ, Kioko T, Bueno EC. Imunoensaios: fundamentos e aplicações. Rio de Janeiro: Guanabara Koogan; 2007.

21. Gonçalves J, Primo CC, Rabbi GMS, Castro DS. Perfil epidemiológico dos casos de sífilis congênita de um hospital universitário - 2004 a 2008. Rev Bras Pesqui Saúde [serial on the internet]. 2011 [cited 2016 Nov 20];13(2):49-55. Available from: http://periodicos.ufes.br/RBPS/article/ viewFile/1602/1202
22. Carvalho IS, Brito RS. Sífilis congênita no Rio Grande do Norte: estudo descritivo do período 2007-2010. Epidemiol Serv Saúde [serial on the internet]. 2014 [cited 2016 Nov 20];23(2):287-94. Available from: http://www.scielo.br/ $\mathrm{pdf} / \mathrm{ress} / \mathrm{v} 23 \mathrm{n} 2 / 1679-4974-$ ress-23-02-00287.pdf

23. Galatoire PSA, Rosso JA, Sakae TM. Incidência de sífilis congênita nos estados do Brasil no período de 2007 a 2009. Revista da Associação Catarinense de Medicina [serial on the internet]. 2012 [cited 2016 Nov 20];41(2):26-32. Available from: http://www.acm.org.br/revista/pdf/artigos/924.pdf

24. Campos ALA, Araújo MAL, Melo SP, Gonçalves MLC. Epidemiologia da sífilis gestacional em Fortaleza, Ceará, Brasil: um agravo sem controle. Cad Saúde Pública [serial on the internet]. 2010 [cited 2016 Nov 20];26(9):1747-55. Available from: http://www.scielo.br/pdf/csp/v26n9/08.pdf

25. Holanda MTCG, Barreto MA, Machado KMM, Pereira RC. Perfil epidemiológico da sífilis congênita no Município do Natal, Rio Grande do Norte-2004 a 2007. Epidemiol Serv Saúde [serial on the internet]. 2011 [cited 2016 Nov 20];20(2):203-12. Available from: http://scielo.iec.pa.gov. br/pdf/ess/v20n2/v20n2a09.pdf

26. Costa CC, Freitas LV, Sousa DMN, Oliveira LL, Chagas ACMA, Lopes MV0, et al. Sífilis congênita no Ceará: analise de uma década. Rev Esc Enferm USP [serial on the internet]. 2013 [cited 2016 Nov 20];47(1):152-9. Available from: http://www.scielo.br/pdf/reeusp/v47n1/a19v47n1.pdf

27. Soeiro CMO, Miranda AE, Saraceni V, Santos MC, Talhari $S$, Ferreira LCL. Syphilis in pregnancy and congenital syphilis in Amazonas State, Brazil: an evaluation using database linkage. Cad Saúde Pública [serial on the internet]. 2014 [cited 2016 Nov 20];30(4):715-23. Available from: http:// www.scielosp.org/scielo.php?script=sci_arttext\&pid=S0102311X2014000400715

Recebido em 13/03/2017 Aprovado em 19/09/2017
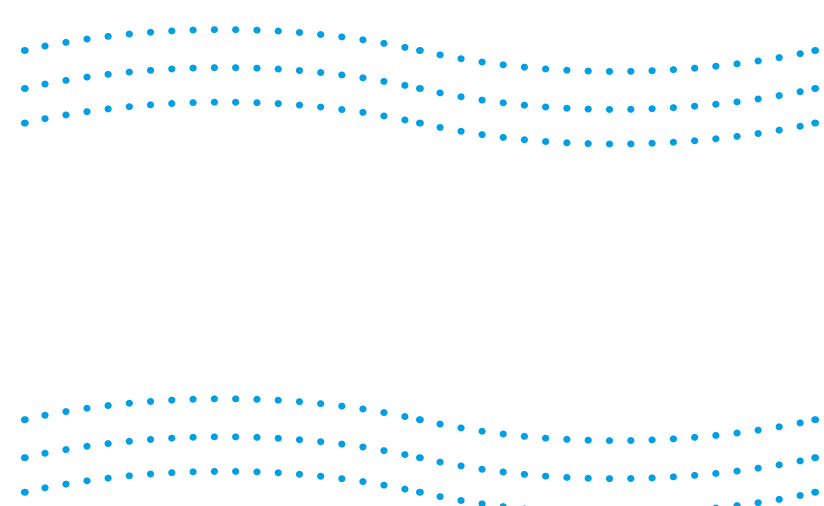

$\ldots \ldots \ldots \ldots \ldots \ldots \ldots$

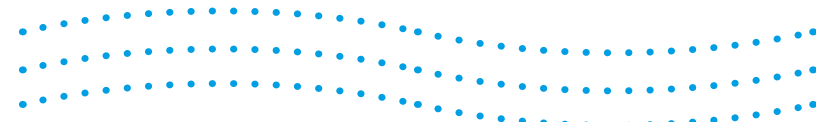

SANARE, Sobral - V.16 n.02,p.51-59, Jul./Dez. - 2017 - 59 